\title{
Primary Cutaneous Aspergillosis Presenting as a Multiloculated Cyst in the Hand
}

\author{
Se Ri Kang ${ }^{1}$, Hong Je Kang ${ }^{2}$, Seon Kwan Juhng ${ }^{1, *}$, Hyun Kyu Yu ${ }^{2}$ and Heon Soo Kim ${ }^{3}$ \\ ${ }^{1}$ Department of Radiology, Wonkwang University School of Medicine, Iksan, Republic of Korea \\ ${ }^{2}$ Department of Orthopedic Surgery, Wonkwang University School of Medicine, Iksan, Republic of Korea \\ ${ }^{3}$ Department of Pathology, Wonkwang University School of Medicine, Iksan, Republic of Korea \\ "Corresponding author: Department of Radiology, Wonkwang University School of Medicine, 54538, Iksan, Republic of Korea. Tel: +82-638591920, Email: \\ juhngsk@wonkwang.ac.kr
}

Received 2019 July 09; Accepted 2019 October 30.

\begin{abstract}
Invasive aspergillosis typically occurs in immunocompromised patients and results in fatal complications. Musculoskeletal involvement of aspergillosis is extremely rare, therefore, when it occurs, its diagnosis and treatment could be delayed. In this paper, we report a rare case of primary invasive aspergillosis with radiologic findings in a patient with rheumatoid arthritis. A 57-yearold woman presented a palpable mass on the right hand with a previous medical history of long-term use of immunosuppressive agents due to rheumatoid arthritis. Magnetic resonance imaging showed a multilobulated and multiloculated cystic lesion with an enhancing wall. The wall of the cystic lesion showed low signal intensity on $\mathrm{T} 2$ weighted images and intermediate to high signal intensity on $\mathrm{T} 1$ weighted images. The mass was confirmed as chronic granulomatous inflammation with multiple acute branching and septated fungal hyphae, consistent with invasive aspergillosis. When a superficial multicystic mass in the hand of especially immunocompromised patients is encountered, invasive aspergillosis should be included in the differential diagnosis, although it is rare.
\end{abstract}

Keywords: Aspergillosis, Hand, Rheumatoid Arthritis

\section{Introduction}

Invasive aspergillosis is typically an opportunistic infection in immunocompromised patients with neutropenia, glucocorticoid therapy, hematopoietic stem cell or solid-organ transplantation $(1,2)$. Aspergillosis manifests as a pulmonary infection in the majority of cases because Aspergillus spores are inhaled regularly $(3,4)$. However, aspergillosis affecting the musculoskeletal system is rare and aspergillosis in hand is extremely rare.

In this report, primary aspergillosis in the hand of a 57year-old rheumatoid arthritis patient with long-term use of immunosuppressive will be discussed and radiologic findings will be described.

\section{Case Presentation}

A 57-year-old female patient presented with a 2-month history of a palpable mass on her right hand, dorsal surface. She had a history of taking steroids and methotrexate for 14 years due to rheumatoid arthritis. The patient had no history of trauma to her right hand and did not mention any other significant medical history, such as venous puncture.

In the physical examination, an approximately $10 \times 5$ cm sized soft palpable mass was revealed on the dorsal aspect of the right fourth proximal phalanx and metacarpal area and the patient did not complain of any pain. Purulent discharge was found on the dorsal aspect of the fourth finger (Figure 1). However, neither localized warmth nor generalized fever was noted. Laboratory testing showed increased leukocyte count (12930/ul) with a normal range of erythrocyte sedimentation rate (ESR) $(5 \mathrm{~mm} / \mathrm{hr}$ ) and Creactive protein $(1.34 \mathrm{mg} / \mathrm{L})$.

Hand radiographs showed a lobulated soft tissue mass with a broad attachment on the dorsal side of the right 4 th proximal phalanx and metacarpal bone with no evidence of abnormal bony findings such as a periosteal reaction was shown (Figure 2). In addition, severe joint space narrowing and osteopenia were found in the wrist joint due to rheumatoid arthritis. Magnetic resonance (MR) imaging revealed a multilobulated and multiloculated cystic lesion on the dorsal side of subcutaneous fat tissue of the 


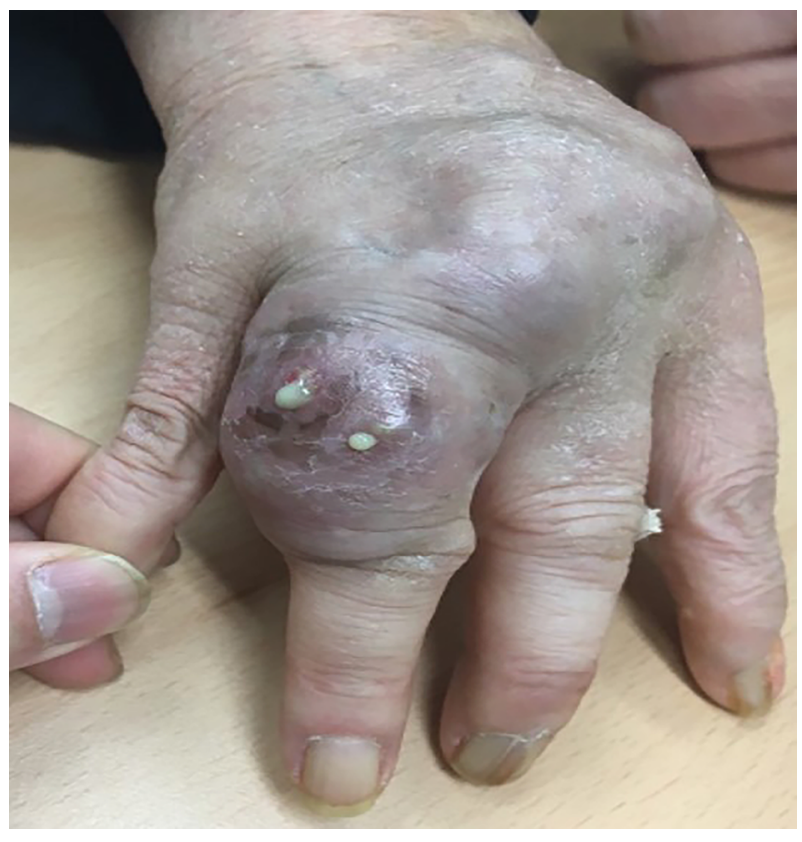

Figure 1. A 57-year-old female patient with a 2-month history of a palpable mass on the dorsal surface of the right hand. Photograph shows an approximate $10 \times 5 \mathrm{~cm}$ sized soft palpable mass on the dorsal aspect of the right 4 th proximal phalanx and metacarpal area with purulent discharge.

fourth metacarpal bone and proximal phalanx. The wall of the cystic lesion showed uniform low signal intensity on T2 weighted images and intermediate to high signal intensity on T1 weighted images (Figure 3). On enhanced T1 weighted images, the wall of the cystic lesion showed diffuse enhancement, but the central portion of the lesion was not distinctly enhanced. The surrounding soft tissue also showed diffuse enhancement. On the other hand, there was no intraarticular or intraosseous extension of the lesion.

The cystic lesion was completely removed by an orthopedic surgeon and operative findings reported a thickwalled multiloculated cyst with abundant odorless sticky yellow pus. Postoperative bacterial cultures were negative, and acid-fast bacillus stain, culture and polymerase chain reaction for mycobacterium tuberculosis were also negative. Histopathology of the lesion showed chronic granulomatous inflammation with multiple acute branching, septated fungal hyphae, consistent with invasive aspergillosis (Figure 4). Chest computed tomography (CT) showed no evidence of pulmonary aspergillosis. The patient was treated with a daily dose of $200 \mathrm{mg}$ voriconazole for 1 month and completely healed without complications.

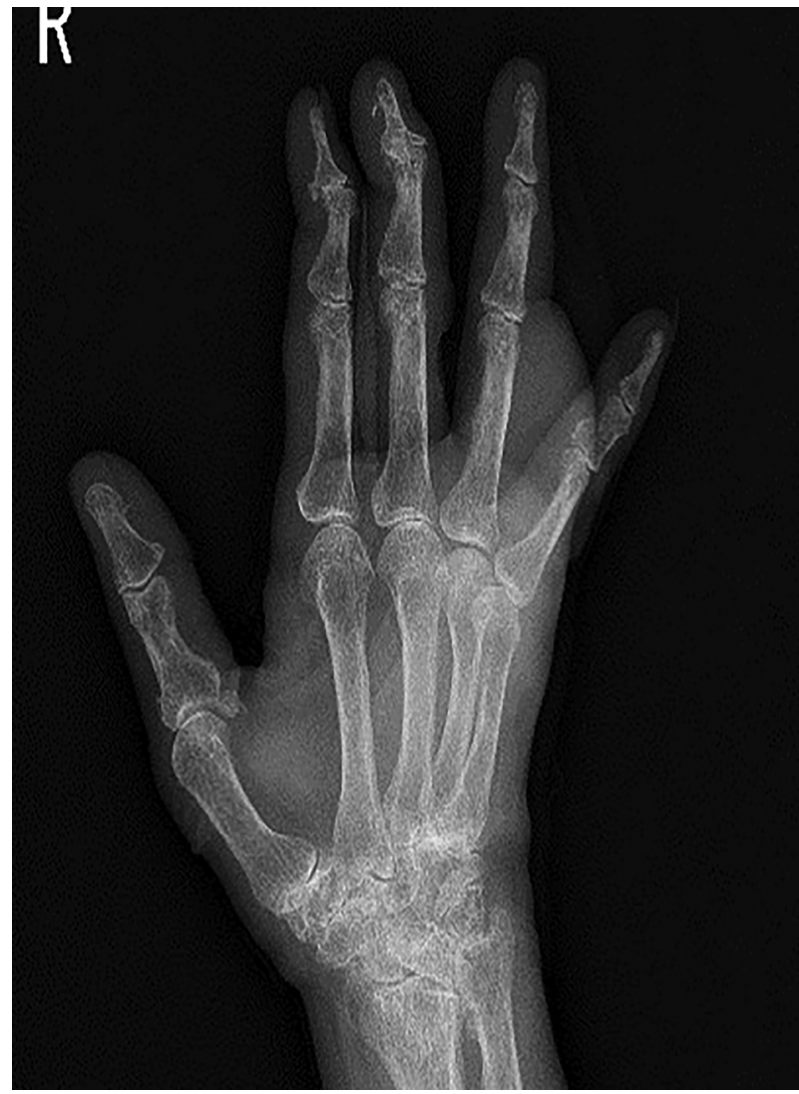

Figure 2. Hand plain radiograph shows a lobulated soft tissue mass on the dorsa aspect of the right 4 th proximal phalanx and metacarpal bone without bony erosion or bony remodeling. Severe joint space narrowing and osteopenia are also found in the wrist joint due to rheumatoid arthritis.

\section{Discussion}

Primary cutaneous aspergillosis has been a rarely reported and suggested pathophysiology that requires a destruction of both mechanical and immunologic barriers (5). As part of the immunologic defense mechanism, macrophages destroy Aspergillus conidia, and polymorphonuclear leukocytes and monocytes ruin Aspergillus hyphae through oxidate and nonoxidative process (5). Additionally, keratin and epidermal layer play the mechanical defense barrier role (5). Accordingly, the organism enters the injured skin by burns, surgical wounds, or sites of intravenous injection or catheter insertion (6). In this case, she denied any history of skin injury in the right hand. However, there could have been unnoticeable microtrauma. In addition, the patient had no any other symptom corresponding to pulmonary involvement or systemic pathology, and her chest CT exhibited no evidence of aspergillosis. These indirectly reflect primary cutaneous infection. 

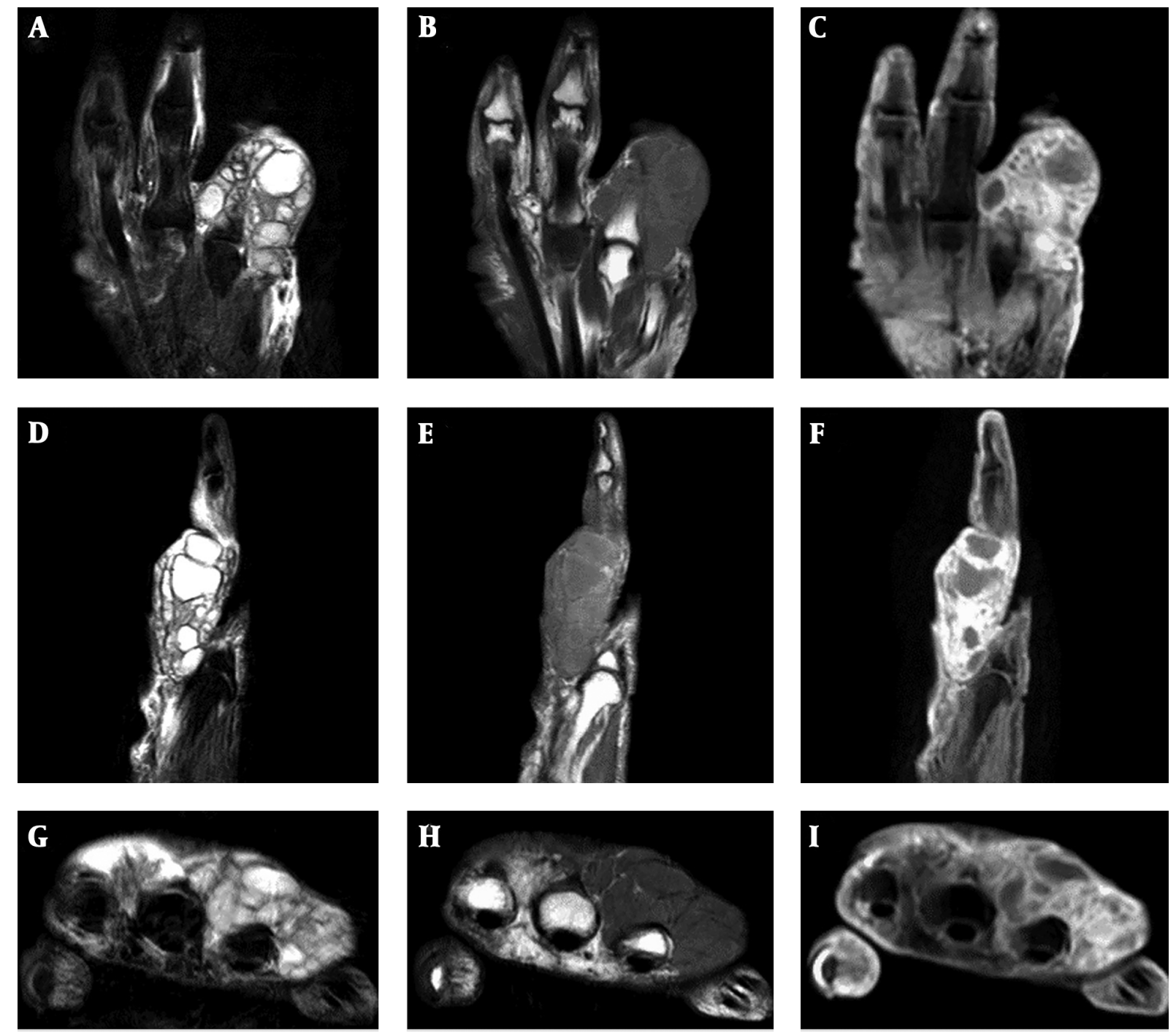

Figure 3. Magnetic resonance imaging, $\mathrm{T} 2(\mathrm{~A}, \mathrm{D}, \mathrm{G})$ and $\mathrm{T} 1 \mathrm{~B}, \mathrm{E}, \mathrm{H})$ weighted images show a multilobulated and multiloculated cystic lesion with central T2 high and T1 low signal intensity. The wall of the cystic lesion shows uniform low signal intensity on T2 weighted images and intermediate to high signal intensity on T1 weighted images. Gadolinium enhanced fat suppressed T1 weighted images (C, F, I) show rim enhancement of the cystic wall and mild enhancement of the surrounding soft tissue. There was no connection between the cystic lesion and adjacent joints.

The cases of primary invasive aspergillosis of the hand have been documented in a few literatures and all reported patients were young children with malignant hematologic disease (3, 7-9). Primary aspergillosis in the hand of a rheumatoid arthritis patient with long-term use of immunosuppressive agents has never been reported yet. This patient has been taking steroid and methotrexate for a long time since diagnosed with rheumatoid arthritis 14 years ago and following immunodeficiency might attribute to aspergillosis.

Cutaneous lesions associated with Aspergillus typically present as erythema and induration such as in our patient, and it often resembles a hemorrhagic blister (3, 6). Hyphal invasion in an immunocompromised patient may lead to angioinvasion that induces ulcer and necrosis, further extension to adjacent osseous structure and soft tissue, and even hematogeneous systemic dissemination that could involve any organ $(5,6)$. Therefore, rapid diagnosis and treatment is important because poorly controlled infection may require amputation of the hand or aggressive systemic therapy, although early definitive diagnosis of apsergillosis remains difficult due to its nonspecific manifestations.

Although imaging findings of primary cutaneous as- 


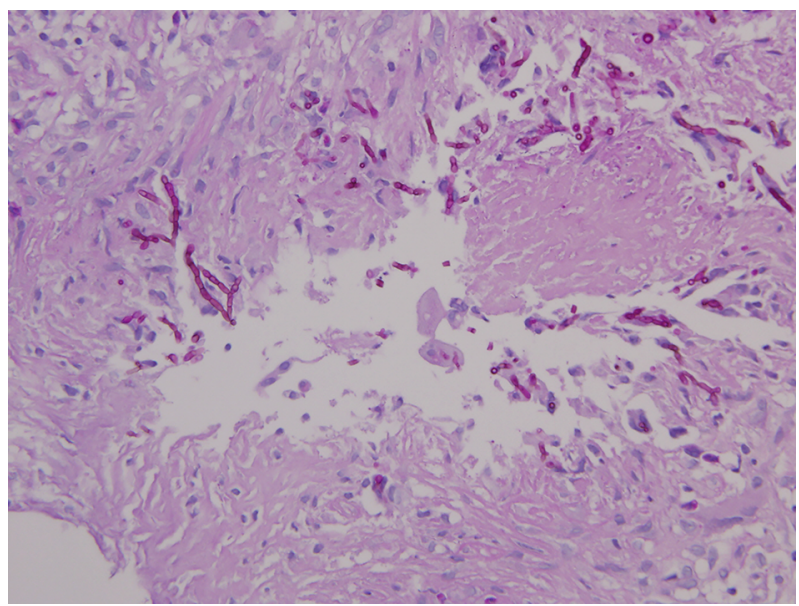

Figure 4. Microscopic examination shows chronic granulomatous inflammation with abscess formation and many acute branching, and septated fungal hyphae. These findings are consistent with invasive aspergillosis (periodic acid-Schiff stain, $\times 400)$.

pergillosis of the hand has never been described, a multilobulated cystic lesion with diffuse enhancement of cystic walls and surrounding soft tissue on MR imaging gave the impression of infected multiple ganglion cysts in our case (10). In addition, rheumatoid nodule occurring in $20 \%$ $30 \%$ of cases diagnosed with rheumatoid arthritis could be typically seen over the bony prominence or extensor surface of the upper extremity with possible combined infection (11). However, this patient presented no communication between the lesion and the joint capsule which could be often observed in cases of ganglion cysts or rheumatoid nodules $(10,11)$. Besides, rheumatoid nodule could be seen as low signal intensity on both $\mathrm{T} 1$ and $\mathrm{T} 2$ weighted images. Thus, it needs to be differentiated from the nodule showing central necrosis. Clinically, tuberculous infection was also suspected due to soft tissue mass containing cold abscess in the background of high endemicity of tuberculosis. Differential diagnosis between Aspergillus infection and tuberculous infection is hard, but the shape of the abscesses could be helpful. Aspergillus abscesses tend to have an irregular and thick wall, while, tuberculous abscesses usually have smooth and thin walls $(12,13)$. The wall of this lesion was thin and smooth, which was not correlated with previous report. However, central T2 hyperintensity of the lesion might be helpful in ruling out tuberculous infection because central caseous necrosis of tuberculous infection is mostly seen as isointensity or hypointensity on $\mathrm{T} 1$ and $\mathrm{T} 2$ weighted images $(14,15)$. The wall of the cystic lesion showed low signal intensity on T2 weighted images and intermediate to high signal intensity on T1 weighted images. Manganese, iron and magnesium in the fungal con- cretions may contribute to the low signal intensity on T2 weighted images, a finding similar to Aspergillus sinusitis and spondylitis $(12,16)$. Areas of high signal intensity on T1 weighted images may be found in Aspergillus abscesses as in our case because of the presence of iron, manganese, or methemoglobin in the lesion (16). Also, hemorrhage occurs frequently in the lesion approximately up to $25 \%$, which could shorten the $\mathrm{T} 1$ relaxation times (16). Besides, multiloculated cystic lesion with enhancement of its wall and the adjacent soft tissue could be considered as infected hydatid cyst caused by Echinococcus. However, its soft tissue involvement is extremely rare as presented as $2.3 \%$ of patients living in endemic areas, and it also shows affinity for muscles at the root of the limbs due to its increased vascularity and decreased activity (17). This patient showed no evidence of daughter cell, floating membrane, or calcified wall that could be presented in case of echinococcosis.

This case is a rare case of primary invasive aspergillosis of the hand in a patient with long term use of an immunosuppressive agent. Invasive aspergillosis has a potential to induce catastrophic progress. Therefore, when a superficial multicystic mass in the hand of especially immunocompromised patients is encountered, invasive aspergillosis should be included in the differential diagnosis, although it is rare.

\section{Footnotes}

Authors' Contributions: Se Ri Kang and Hong Je Kang wrote the manuscript. Seon Kwan Juhng contributed to the basic concept of the report and proofread the manuscript. Hyun Kyu Yu supervised and reviewed the manuscript. Heon Soo Kim reviewed all the pathologic slides and provided pathologic images.

Conflict of Interests: The authors declare that they have no conflict of interest and no financial interests related to the material in the manuscript.

Ethical Approval: Patient informed consent was obtained. This manuscript was approved by our institutional review board.

Funding/Support: This paper was supported by Wonkwang University in 2018.

\section{References}

1. Montejo M. [Epidemiology of invasive fungal infection in solid organ transplant]. Rev Iberoam Micol. 2011;28(3):120-3. Spanish. doi: 10.1016/j.riam.2011.06.003. [PubMed: 21700231].

2. Dal T, Tekin A, Tekin R, Deveci Ö, Frrat U, Mete M, et al. Soft tissue abscess caused by aspergillus fumigatus in an immunosuppressive patient. Euro J General Med. 2013;10(2):118-22. doi: 10.29333/ejgm/82292. 
3. Jones NF, Conklin WT, Albo VC. Primary invasive aspergillosis of the hand. J Hand Surg Am. 1986;11(3):425-8. doi: 10.1016/s03635023(86)80156-3. [PubMed: 3086427].

4. Segal BH. Aspergillosis. N Engl J Med. 2009;360(18):1870-84. doi: 10.1056/NEJMra0808853. [PubMed: 19403905].

5. Liu X, Yang J, Ma W. Primary cutaneous aspergillosis caused by Aspergillus.fumigatus in an immunocompetent patient: A case report. Medicine (Baltimore). 2017;96(48). e8916. doi: 10.1097/MD.0000000000008916. [PubMed: 29310381]. [PubMed Central: PMC5728782].

6. Olorunnipa O, Zhang AY, Curtin CM. Invasive aspergillosis of the hand caused by aspergillus ustus: A case report. Hand (NY). 2010;5(1):102-5. doi: 10.1007/s11552-009-9211-x. [PubMed: 19568818]. [PubMed Central: PMC2820612].

7. Young RC, Bennett JE, Vogel CL, Carbone PP, DeVita VT. Aspergillosis. The spectrum of the disease in 98 patients. Medicine (Baltimore). 1970;49(2):147-73. doi: 10.1097/00005792-197003000-00002. [PubMed: 4913991].

8. Prystowsky SD, Vogelstein B, Ettinger DS, Merz WG, Kaizer H, Sulica VI, et al. Invasive aspergillosis. N Engl J Med. 1976;295(12):655-8. doi: 10.1056/NEJM197609162951206. [PubMed: 823431].

9. Goldberg B, Eversmann WW, Eitzen EJ. Invasive aspergillosis of the hand. J Hand Surg Am. 1982;7(1):38-42. doi: 10.1016/s03635023(82)80011-7. [PubMed: 6949983].

10. Rao A, Nandikoor S, Mallarajapatna G, Meghanathan P. Unusual presentation of an usual condition-cystic ganglionosis. BJR Case Rep.
2016;2(1):20150231. doi: 10.1259/bjrcr.20150231. [PubMed: 30364382]. [PubMed Central: PMC6195920].

11. Sanders TG, Linares R, Su A. Rheumatoid nodule of the foot: MRI appearances mimicking an indeterminate soft tissue mass. Skeletal Radiol. 1998;27(8):457-60. doi: 10.1007/s002560050418. [PubMed: 9765141].

12. Kwon JW, Hong SH, Choi SH, Yoon YC, Lee SH. MRI findings of Aspergillus spondylitis. AJR Am J Roentgenol. 2011;197(5):W919-23. doi: 10.2214/AJR.11.6786. [PubMed: 22021542].

13. Jung NY, Jee WH, Ha KY, Park CK, Byun JY. Discrimination of tuberculous spondylitis from pyogenic spondylitis on MRI. AJR Am J Roentgenol. 2004;182(6):1405-10. doi: 10.2214/ajr.182.6.1821405. [PubMed: 15149983].

14. Kim TK, Chang KH, Kim CJ, Goo JM, Kook MC, Han MH. Intracranial tuberculoma: Comparison of MR with pathologic findings. AJNR Am J Neuroradiol. 1995;16(9):1903-8. [PubMed: 8693993].

15. Ahn T, Juhng SK, Mok Lee G, Soo Kim H. Musculoskeletal tuberculosis presenting as isolated tuberculous gumma in an immunocompetent patient. Iran J Radiol. 2018;15(2). e59213. doi:10.5812/iranjradiol.59213.

16. Almutairi BM, Nguyen TB, Jansen GH, Asseri AH. Invasive aspergillosis of the brain: Radiologic-pathologic correlation. Radiographics. 2009;29(2):375-9. doi: 10.1148/rg.292075143. [PubMed: 19325053].

17. Polat P, Kantarci M, Alper F, Suma S, Koruyucu MB, Okur A. Hydatid disease from head to toe. Radiographics. 2003;23(2):475-94. quiz 5367. doi: 10.1148/rg.232025704. [PubMed: 12640161]. 